\title{
Effects of swimming exercise on social isolation-induced memory impairment and apoptosis in old rats
}

\author{
Sang-Seo Park', Hye-Sang Park' ${ }^{2}$ Tae-Woon Kim ${ }^{1,3}$, Sam-Jun Lee $e^{4, *}$ \\ 'Department of Physiology, College of Medicine, Kyung Hee University, Seoul, Korea \\ ${ }^{2}$ Department of Kinesiology, College of Public Health and Cardiovascular Research Center, Lewis Katz School of Medicine, Temple University, Philadelphia, PA, USA \\ ${ }^{3}$ Exercise Rehabilitation Research Institute, Department of Exercise \& Health Science, Sangmyung University, Seoul, Korea \\ ${ }^{4}$ Department of Sport Rehabilitation, Tongmyong University, Busan, Korea
}

Effect of swimming exercise on serotonin (5-hydroxytryptamine, 5-HT) expression and apoptosis in social isolation rats during old age was investigated. Rats in the old social isolation groups were housed alone per cage for 4 weeks. Rats in the swimming exercise groups were allowed to swim for 30 min once daily for 4 weeks. Morris water maze task determined spatial working memory and elevated plus maze test determined anxiety. Immunohistochemistry for tryptophan hydroxylase (TPH) and 5-HT in the dorsal raphe and for doublecortin (DCX) in the hippocampal dentate gyrus was conducted. Terminal deoxynucleotidyl transferase-mediated dUTP nick end labeling (TUNEL) staining in the hippocampal dentate gyrus was performed. Western blot analysis for Bax, Bcl-2, and cytochrome $\mathrm{c}$ in the hippocampus was conducted. Social isolation in rats of old age reduced spatial working memory and increased anxiety level. Swimming exercise enhanced spatial working memory and suppressed anxiety level. Social isolation in rats of old age inhibited TPH and 5-HT expression in dorsal rape. Swimming exercise increased TPH and 5-HT expression. Social isolation in rats of old age inhibited DCX-positive cells in the hippocampal dente gyrus. Swimming exercise increased DCX-positive cells. Social isolation in rats of old age increased TUNEL-positive cells, Bax and cytochrome c expression, and decreased Bcl-2 expression, which promoted apoptosis. Swimming exercise suppressed TUNEL-positive cells, Bax and cytochrome $c$ expression, and increased Bcl-2 expression, which inhibited apoptosis. Swimming exercise improved 5-HT expression and suppressed apoptosis to alleviate anxiety and memory impairment during old age.

Keywords: Social isolation, Anxiety, Spatial working memory, Apoptosis, Swimming exercise

\section{INTRODUCTION}

Social isolation is associated with an increased risk for depressive symptoms, suicide attempts, and low self-esteem (Hall-Lande et al., 2007). Long-term social isolation can lead to serious morbidity, difficulty, dissatisfaction, and aggression. Social isolation leads to a decrease in memory, promotes the expression of depression-related factors, and inhibits neuronal production (Huang et al., 2011).

Serotonin (5-hydroxytryptamine, 5-HT) is a representative monoamine neurotransmitter and is synthesized from tryptophan, which is regulated by tryptophan hydroxylase (TPH) (Goridis and Rohrer, 2002). Various factors cause depression, but monoamine neurotransmitters such as dopamine and serotonin play an important role

(Pittenger and Duman, 2008). 5-HT and TPH are involved in the mood regulation (Park et al., 2019). Stress causes neuronal cell death and neuroplastic disorders, which are closely related to cognitive decline and anxiety (Bannerman et al., 2014).

The hippocampus is an important area of the brain that is involved in the process of learning and memory formation, and hippocampal nerve production continues to occur after the development of the nervous system (López-Toledano and Shelanski, 2004). Doublecortin (DCX) is a marker of neuronal progenitor cells, and DCX is involved in neuronal migration and development (Lee et al., 2018; Park et al., 2019).

Apoptosis is a form of cell death that serves to remove dying cells, and therefore apoptosis plays an important role in normal

${ }^{*}$ Corresponding author: Sam-Jun Lee (iD https://orcid.org/0000-0002-8491-3602 Department of Sport Rehabilitation, College of Health, Social Welfare, and Education, Tongmyong University, 428 Sinseonno, Nam-gu, Busan 48520, Korea E-mail: anada23@tu.ac.kr

Received: April 15, 2020 / Accepted: May 20, 2020 tribution Non-Commercial License (https://creativecommons.org/licenses/by-nc/4.0/) which permits unrestricted non-commercial use, distribution, and reproduction in any medium, provided the original work is properly cited. 
development and tissue homeostasis. Nevertheless, inappropriate or excessive apoptosis is associated with various neurological disorders (Lee et al., 2003; Song et al., 2018). Bcl-2 family is classified according to its function as antiapoptotic proteins and proapoptotic proteins, and then determines the mitochondrial response to apoptotic stimuli (Upadhyay et al., 2003). Bcl-2 can inhibit apoptosis by preventing the release of cytochrome $\mathrm{c}$ from mitochondria. However, $\mathrm{Bcl}-2$ and $\mathrm{Bcl}-\mathrm{xL}$ form heterodimers with the major pro-apoptotic members Bax and lose their ability in their preventive function (Kuwana and Newmeyer, 2003; Song et al., 2018). Terminal deoxynucleotidyl transferase-mediated dUTP nick end labeling (TUNEL) analysis detects DNA fragmentation, one of the hallmarks of apoptotic cell death (Song et al., 2018).

Exercise is a leading factor in improving brain function, and facilitates the signaling of monoamine neurotransmitters (Daley, 2008). Exercise plays a role in regulating signaling pathways by various neurotransmitters in the hippocampus in relation to neuroplasticity (Staples et al., 2015). The aim of this study was to investigate the effect of swimming exercise on 5-HT expression and apoptosis in social isolation rats during old age.

\section{MATERIALS AND METHODS}

\section{Experiment animals}

Male Wistar rats (15 months old) were used for this experiment. This study was approved by the Kyung Hee University Institutional Animal Care and Use Committee in Seoul, Korea (KHUASP [SE]-16-154). The animals were randomly divided into four groups ( $\mathrm{n}=10$ per group): old control group, old control and swimming exercise group, old social isolation group, old social isolation and swimming exercise group.

\section{Social isolation protocol}

Social isolation was performed according to the Ren et al. (2015). At first, 15 months old rats were adapted to laboratory conditions for a week. The rats in the old social isolation groups were housed alone per cage $(20 \mathrm{~cm} \times 26 \mathrm{~cm} \times 13 \mathrm{~cm})$ for 4 weeks. The rats in the old control groups were housed three rats per cage for 4 weeks.

\section{Swimming exercise protocol}

The rats in the swimming exercise groups were allowed to swim for 30 min once daily, for 5 days per week, during 4 consecutive weeks. Swimming pool $(120 \mathrm{~cm} \times 80 \mathrm{~cm} \times 50 \mathrm{~cm})$ was filled with water at a temperature of $30^{\circ} \mathrm{C} \pm 2^{\circ} \mathrm{C}$. Swimming exercise was performed with weight of $5 \%$ of the body weight.

\section{Morris water maze task}

Spatial working memory was evaluated using the Morris water maze task, as the previous described method (Park et al., 2019). This Morris water task required rats to train their spatial location of hidden platform in circular pool (50-cm height and $180-\mathrm{cm}$ diameter) filled with water $\left(30^{\circ} \mathrm{C} \pm 1^{\circ} \mathrm{C}\right)$. A hidden platform ( $40-\mathrm{cm}$ height and $15-\mathrm{cm}$ diameter) was placed $2 \mathrm{~cm}$ below the water surface in the middle of the north quadrant and disguised as a black background. The learning test consisted of three trials each day for 5 consecutive days.

During each trial, the rat was placed in the water facing the wall of the maze in one of the three quadrants that did not contain the platform. The time spent mounting the platform (escape latency, with a cutoff time of $20 \mathrm{sec}$ ) and the swim speed was recorded. The probe trial $(60 \mathrm{sec})$, in which the platform was removed, was performed $24 \mathrm{hr}$ after the end of the 6 day of training. The percentage of time spent in the target quadrant was considered an indicator of memory performance.

\section{Elevated plus maze test}

In order to evaluate anxiety, elevated plus maze test was performed, as the previous method (Park et al., 2019). The plus maze consisted of black acrylic with two open arms $(50 \mathrm{~cm} \times 10 \mathrm{~cm} \times$ $36 \mathrm{~cm})$ and two closed arms $(50 \mathrm{~cm} \times 10 \mathrm{~cm} \times 36 \mathrm{~cm})$, where two arms met each other and connected to the central platform $(10 \mathrm{~cm} \times$ $10 \mathrm{~cm}$ ). The entire plus maze was raised to a height of $60 \mathrm{~cm}$ from the bottom and illuminated with a 100-W light bulb fixed $2 \mathrm{~m}$ above the bottom of the maze. The rat was placed on the center platform facing a closed arm and allowed to explore the maze freely for $300 \mathrm{sec}$. Entering the arm was defined as putting all 4 feet in the arm. Entry and time in the open arms were measured.

\section{Tissue preparation}

The rats were sacrificed immediately after finishing last behavior test. To prepare the brain slices, the rats were fully anesthetized with Zoletil $50(10 \mathrm{mg} / \mathrm{kg}$, intraperitoneally; Vibac Laboratories, Carros, France). After that, the rats were transcardially perfused with $50 \mathrm{mM}$ phosphate-buffered saline (PBS) and then fixed with freshly prepared $4 \%$ paraformaldehyde in $100 \mathrm{mM}$ phosphate buffer $(\mathrm{pH}, 7.4)$. The brains were then removed, postfixed in the same fixative solution overnight, and transferred into a $30 \%$ sucrose solution for cryoprotection. Coronal sections with thicknesses of $40 \mu \mathrm{m}$ were made using a freezing microtome (Leica, Nussloch, Germany). 


\section{Immunohistochemistry for TPH and 5-HT}

As previously described method (Park et al., 2019), immunohistochemistry for TPH and 5-HT in the dorsal raphe was performed. The sections were incubated in PBS for $10 \mathrm{~min}$, and then washed 3 times in the same buffer. The sections were then incubated in $1 \% \mathrm{H}_{2} \mathrm{O}_{2}$ for $20 \mathrm{~min}$. The sections were selected from each brain and incubated overnight with mouse anti-TPH antibody (Abcam, Cambridge, UK) and rabbit anti-5-HT antibody (Abcam) at a dilution of 1:200 for TPH and 5-HT expression. The sections were incubated for $1 \mathrm{hr}$ with biotinylated anti-mouse secondary antibody or with biotinylated anti-rabbit secondary antibody (Vector Laboratories, Burlingame, CA, USA). They were subsequently incubated with avidin-biotin-peroxidase complex (Vector Laboratories) for $1 \mathrm{hr}$. Immunoreactivity was visualized by incubating the sections with a solution consisting of $0.05 \%$ 3,3'-diaminobenzidine and $0.01 \% \mathrm{H}_{2} \mathrm{O}_{2}$ in $50 \mathrm{mM}$ Tris-buffer
$(\mathrm{pH}, 7.6)$ for approximately 3 min. The sections were finally mounted on gelatin-coated glass slides. The slides were air-dried overnight at room temperature, and the coverslips were mounted using Permount (Thermo Fisher Scientific Inc., Waltham, MA, USA).

\section{Immunohistochemistry for DCX}

As previously described method (Park et al., 2019), immunohistochemistry for DCX in the dorsal raphe were performed. The sections were incubated with PBS for $10 \mathrm{~min}$, washed 3 times in PBS, and then incubated with $1 \% \mathrm{H}_{2} \mathrm{O}_{2}$ for $30 \mathrm{~min}$. The sections were incubated overnight with goat anti-DCX antibody (1:1,000; Abcam). They were then incubated with the appropriate biotinylated secondary antibody (1:500; Vector Laboratories) for another 1 hour, washed, and incubated in ABC complex kit (1:100; Vector Laboratories). Labeling was visualized using $0.03 \%$ diami-
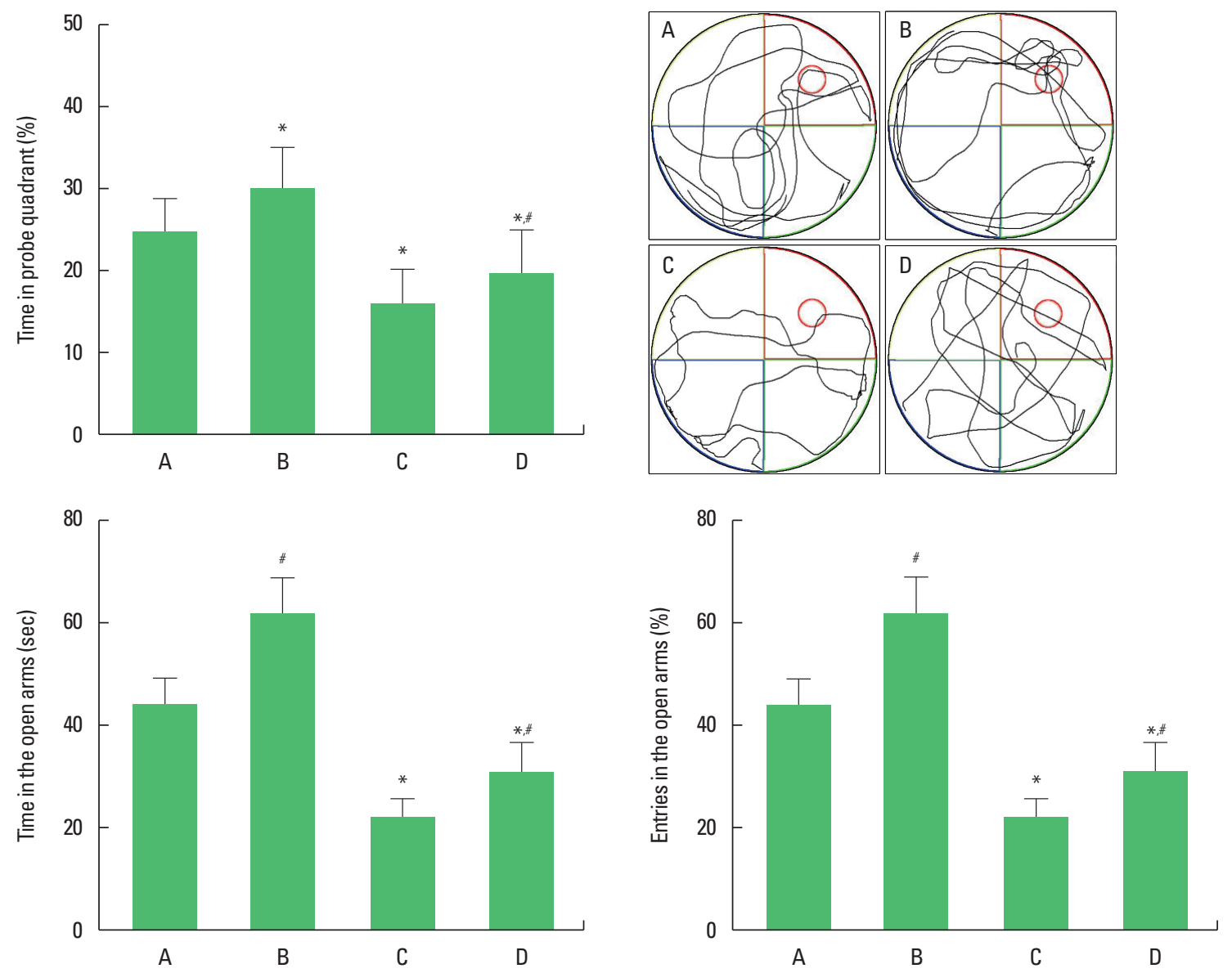

Fig. 1. The effects of exercise on the spatial working memory in water maze test (upper panel) and anxiety in elevated plus maze test (lower panel). A, old control group; B, old control and swimming exercise group; $C$, old social isolation group; D, old social isolation and swimming exercise group. The data are presented as the mean \pm standard error of the mean. ${ }^{*} P<0.05$ compared to the old control group. ${ }^{*} P<0.05$ compared to the old social isolation group. 
nobenzidine, and the sections were mounted onto gelatin-coated slides. The slides were air-dried overnight at room temperature, and the coverslips were mounted using Permount (Thermo Fisher Scientific Inc.).

\section{TUNEL staining}

As previously described method (Song et al., 2018), we performed TUNEL staining using a Cell Death Detection Kit (Roche, Mannheim, Germany). The sections were postfixed in ethanol-acetic acid (2:1), rinsed, incubated with proteinase K (100 $\mathrm{mg} / \mathrm{mL}$ ), and rinsed again. They were then incubated in $3 \%$ hydrogen peroxide, permeabilized with $0.5 \%$ Triton X-100, rinsed again, and incubated in the TUNEL reaction mixture. The sections were rinsed and visualized using Converter-POD with $0.03 \%$ diaminobenzidine, counterstained with Nissl and mount- ed onto gelatin-coated slides. The slides were air-dried overnight at room temperature and the coverslips were mounted using Permount (Thermo Fisher Scientific Inc.).

\section{Western blotting for Bax, Bcl-2, and cytochrome c}

As previously described method (Song et al., 2018), western blot was conducted. The hippocampus tissues were homogenized on ice and lysed in a lysis buffer containing $50 \mathrm{mM}$ Tris- $\mathrm{HCl}(\mathrm{pH}$, 7.5), $150 \mathrm{mM} \mathrm{NaCl}, 0.5 \%$ deoxycholic acid, $1 \%$ Nonidet P40, $0.1 \%$ sodium dodecyl sulfate (SDS), $1 \mathrm{mM}$ phenylmethylsulfonyl fluoride, and $100-\mathrm{mg} / \mathrm{mL}$ leupeptin. Protein content was measured using a Bio-Rad colorimetric protein assay kit (Bio-Rad, Hercules, CA, USA). Protein of $40 \mu \mathrm{g}$ was separated on SDS-polyacrylamide gels and transferred onto a nitrocellulose membrane, which was incubated with mouse $\beta$-actin antibody $(1: 3,000$; San-
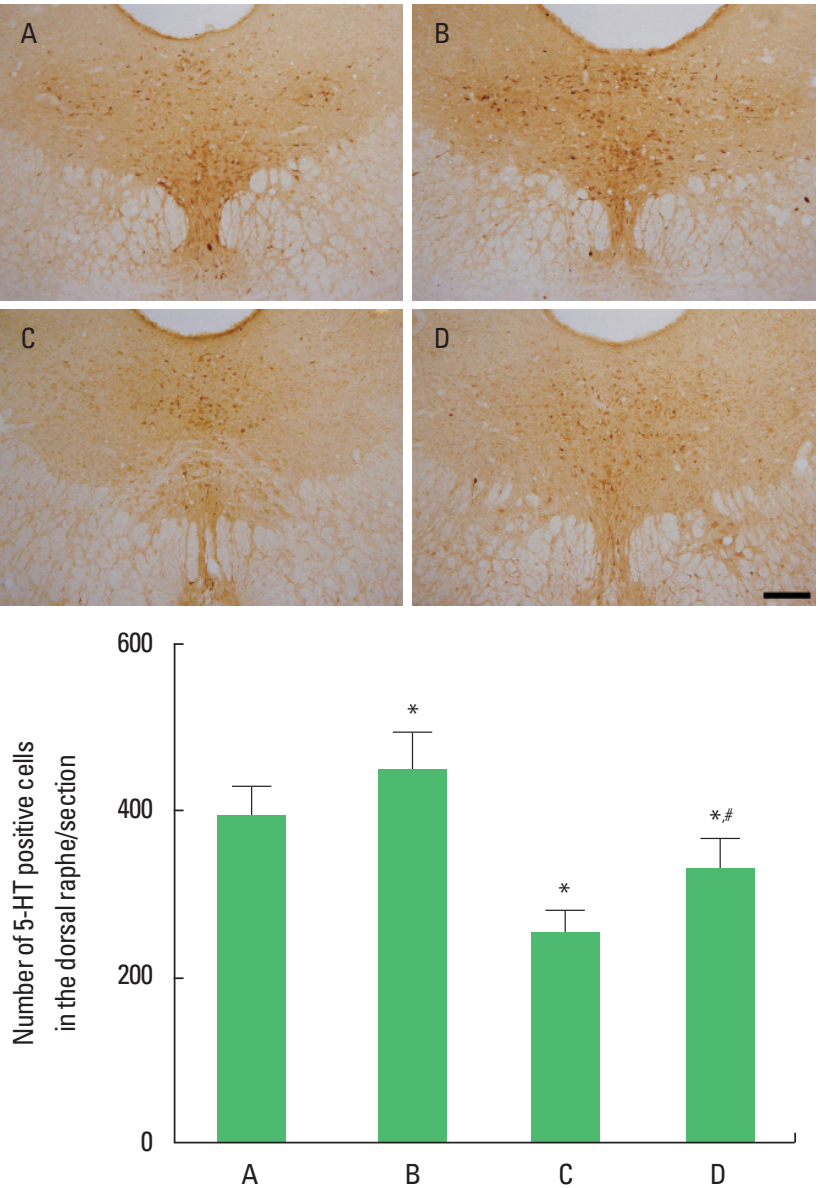

Fig. 2. The effects of exercise on tryptophan hydroxylase (TPH)-positive cells and 5-hydroxytryptamine (5-HT) positive cells in the dorsal raphe. Upper panel: immunohitochemical staining of TPH (left) and 5-HT (right). The scale bar represents $200 \mu \mathrm{m}$. Lower panel: the number of TPH-positive cells (left) and 5-HT-positive cells (right). A, old control group; B, old control and swimming exercise group; C, old social isolation group; D, old social isolation and swimming exercise group. The data are presented as the mean \pm standard error of the mean. ${ }^{*} P<0.05$ compared to the old control group. ${ }^{\sharp} P<0.05$ compared to the old social isolation group. 
ta Cruz Biotechnology, Santa Cruz, CA, USA), mouse Bcl-2 antibody (1:1,000; Santa Cruz Biotechnology), rabbit Bax antibody (1:1,000; Santa Cruz Biotechnology), and rabbit cytochrome c antibody (1:1,000; Cell Signaling Technology, Beverly, MA, USA). Horseradish peroxidase-conjugated anti-mouse for $\beta$-actin and $\mathrm{Bcl}-2$, anti-rabbit for Bax and cytochrome $\mathrm{c}$ were used as secondary antibodies.

\section{Data analysis}

For confirming expression of Bax, Bcl-2, and cytochrome c, the detected bands were calculated densitometrically using Molecular Analyst (version 1.4.1, Bio-Rad). The number of TPH-positive and 5-HT-positive cells in the dorsal raphe and the number of DCX-positive and TUNEL-positive cells in dentate gyrus were counted hemilaterally under a light microscope (Olympus, Tokyo,
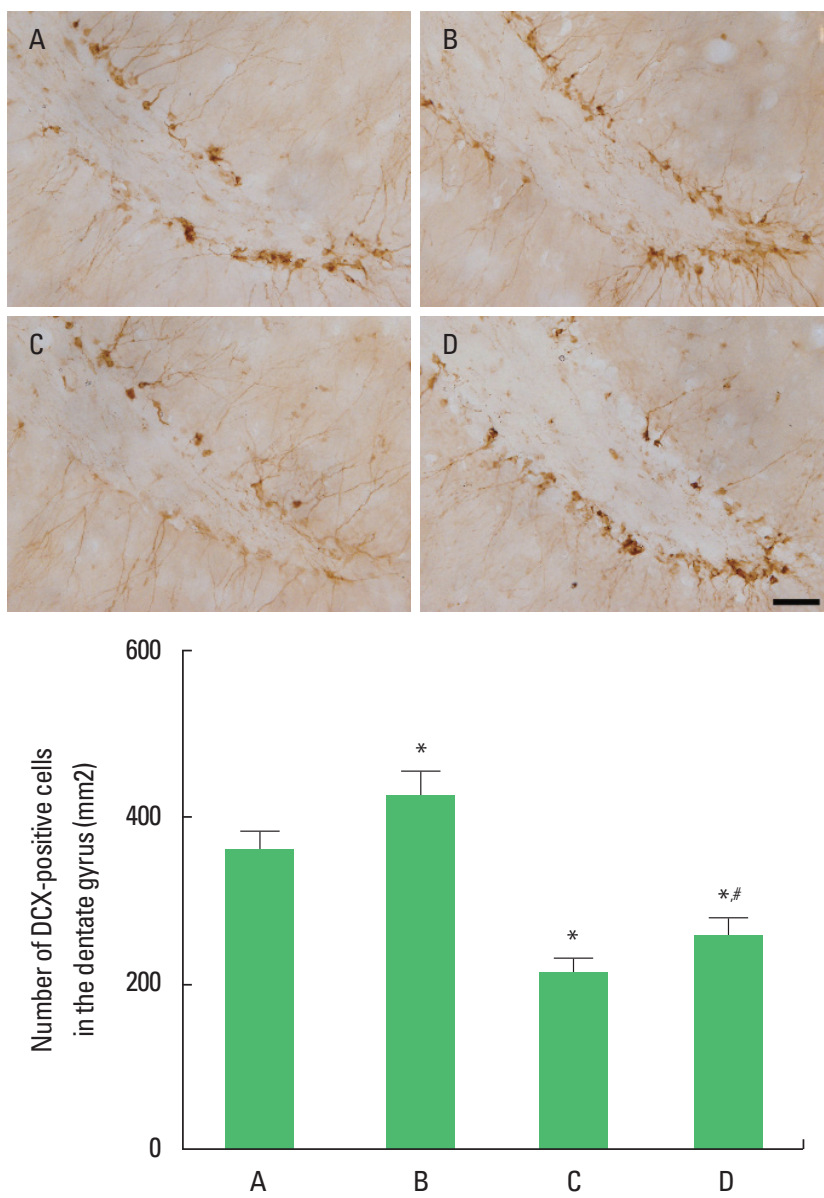

Japan). The data were analyzed with one-way analysis of variance and then Tukey post hoc test. All values are expressed as the mean \pm standard error of the mean, and $P$-value $<0.05$ was considered significant.

\section{RESULTS}

\section{Spatial working memory and anxiety}

The time of passing the probe quadrant was shortened in the rats of the social isolation group $(P<0.05)$. However, swimming exercise lengthened the time of passing the probe quadrant in social isolation rats $(P<0.05)$. The time and $\%$ of entry in the open arms were shortened in the rats of the social isolation group $(P<$ $0.05)$. However, swimming exercise lengthened the time and $\%$ of entry in the open arms in the social isolation rats $(P<0.05)$ (Fig. 1).
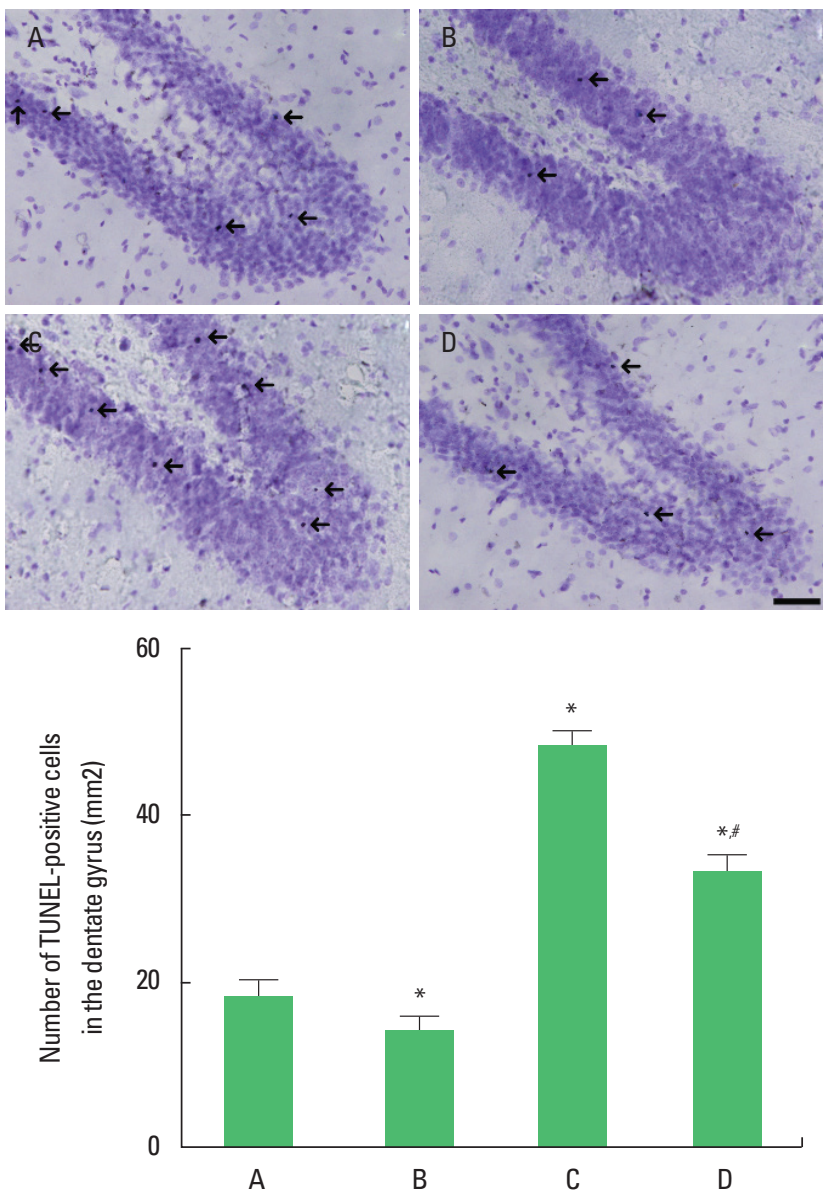

Fig. 3. The effects of exercise on doublecortin (DCX)-positive cells and terminal deoxynucleotidyl transferase-mediated dUTP nick end labeling (TUNEL)-positive cells in the dentate gyrus. Upper panel: immunohitochemical staining of DCX (left) and TUNEL staining (right). The scale bar represents $200 \mu \mathrm{m}$. Arrows indicate TUNEL-positive cells. Lower panel: the number of DCX-positive cells (left) and TUNEL-positive cells (right). A, old control group; B, old control and swimming exercise group; $C$, old social isolation group; $D$, old social isolation and swimming exercise group. The data are presented as the mean \pm standard error of the mean. ${ }^{*} P<0.05$ compared to the old control group. ${ }^{\#} P<0.05$ compared to the old social isolation group. 


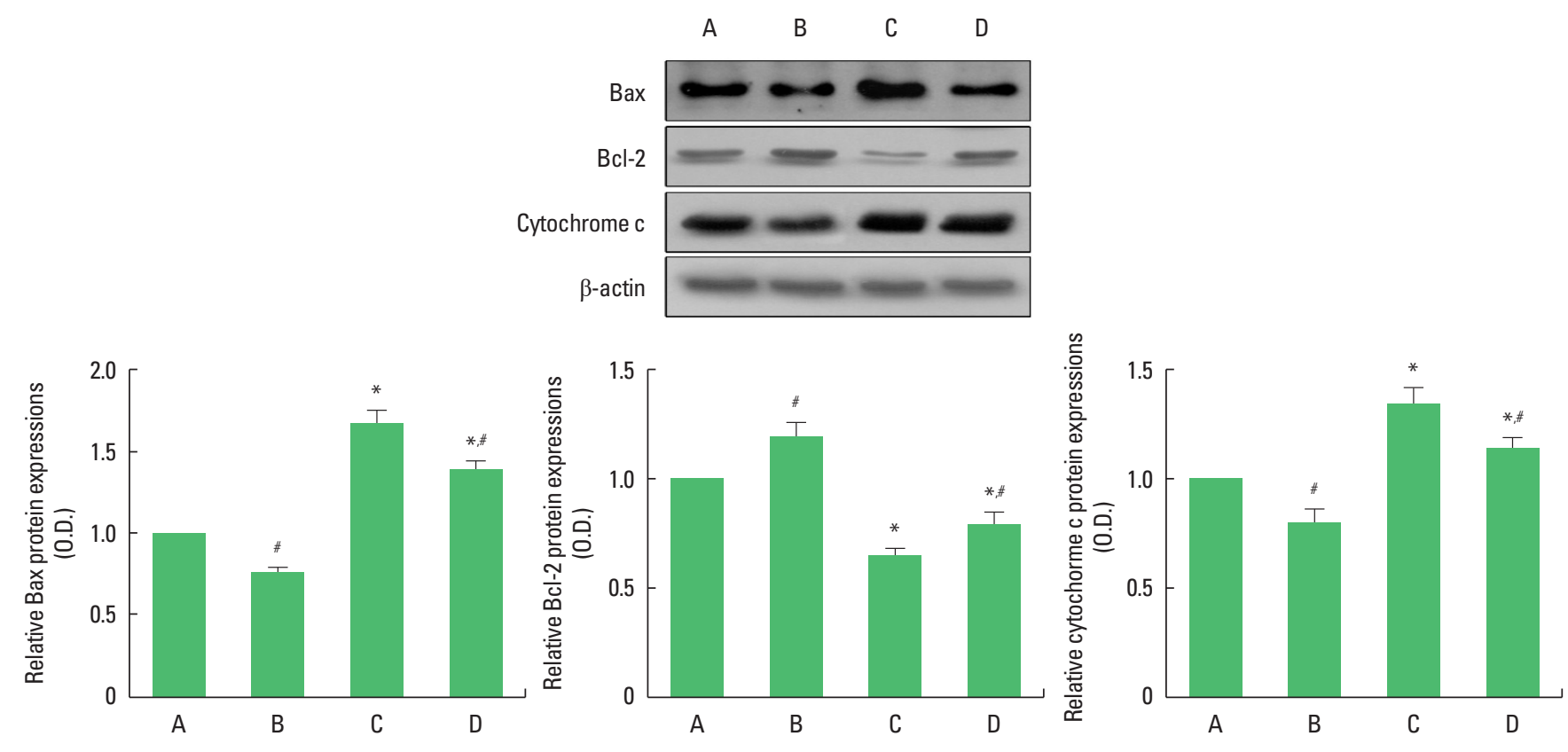

Fig. 4. The effects of exercise on the expressions Bax, Bcl-2, and cytochrome $\mathrm{c}$ in the hippocampus. Upper panel: representative expression of Bax, Bcl-2, and cytochrome c. Lower panel: relative expression of of Bax (left), Bcl-2 (middle), and cytochrome c (right). A, old control group; B, old control and swimming exercise group; C, old social isolation group; $D$, old social isolation and swimming exercise group. The data are presented as the mean \pm standard error of the mean. ${ }^{*} P<0.05$ compared to the old control group. ${ }^{\#} P<0.05$ compared to the old social isolation group.

\section{TPH and 5-HT expression in the dorsal raphe}

The number of TPH-positive and 5-HT-positive cells in the dorsal raphe was decreased in the rats of the social isolation group $(P<0.05)$. However, swimming exercise increased the number of TPH-positive and 5-HT-positive cells in the social isolation rats $(P<0.05)$ (Fig. 2).

\section{DCX-positive and TUNEL-positive cells in the dentate gyrus}

The number of DCX-positive cells in the dentate gyrus was decreased $(P<0.05)$ and the number of TUNEL-positive cells in the dentate gyrus was increased in the rats of the social isolation group $(P<0.05)$. However, swimming exercise increased the number of DCX-positive cells $(P<0.05)$ and suppressed the number of TUNELpositive cells in the social isolation rats $(P<0.05)$ (Fig. 3).

\section{Bax, $\mathrm{BCl}-2$, and cytochrome cexpression in the hippocampus}

Bax and cytochrome $\mathrm{c}$ expression in the hippocampus was increased $(P<0.05)$ and $\mathrm{Bcl}-2$ expression in the hippocampus was decreased in the rats of the social isolation group $(P<0.05)$. However, swimming exercise suppressed $B a x$ and cytochrome $c$ expression $(P<0.05)$ and increased $\mathrm{Bcl}-2$ expression in the social isolation rats $(P<0.05)$ (Fig. 4).

\section{DISCUSSION}

Social isolation burdens a strain on the health and well-being of the old people (Hawton et al., 2011). In this study, Morris water maze task showed that social isolation reduced spatial working memory and elevated plus maze test showed that social isolation increased anxiety level. In contrast, swimming exercise enhanced spatial working memory in Morris water maze task and suppressed anxiety level in elevated plus maze test.

Activation of 5-HT neurons played an important role in behaviors related to depression and anxiety (Teissier et al., 2015). Social isolation reduced the excitability of 5-HT neurons in the dorsal rape (Sargin et al., 2016). In this study, social isolation in rats of old age suppressed TPH and 5-HT expression in dorsal rape. In contrast, swimming exercise increased TPH and 5-HT expression. Increases 5-HT synthesis and activity may be involved in the antidepressant effect of exercise in the old people (Melancon et al., 2012).

Chronic unpredictable stress for 15 days significantly decreased cell proliferation (Banasr et al., 2007). Both male and female rats are susceptible to the suppressive effect of social isolation on exercise-induced neurogenesis (Leasure et al., 2009). Social isolation inhibited neuronal production by promoting the expression of depression-related parameters (Huang et al., 2011). In addition, so- 
cial isolation can inhibit hippocampal neurogenesis and impair adult spatial memory (McCormick et al., 2012). In this study, social isolation in rats of old age suppressed the number of DCXpositive cells in the hippocampal dente gyrus. In contrast, swimming exercise increased the number of DCX-positive cells.

Chronic social separation has led to the onset of synaptic signaling that may be etiologically related to impaired adaptive responses of the central nervous system (Djordjevic et al., 2010). Bcl-2 inhibits apoptosis by regulating the release of cytochrome $\mathrm{c}$ and other proteins from the mitochondria. $\mathrm{Bcl}-2$ functions as an inhibitor of mitochondrial permeability by binding to the membrane-inserted Bax monomer and changing the morphology of the mitochondrial membrane to prevent productive oligomerization of Bax (Dlugosz et al., 2006). Social isolation increased the number of hippocampal TUNEL-positive cells and enhanced expression of cytochrome c, which activated apoptosis (Wang et al., 2019). In this study, the number of TUNEL-positive cells and expression of $\mathrm{Bax}$ and cytochrome $\mathrm{c}$ were increased, while expression of $\mathrm{Bcl}-2$ was decreased in the old rats that underwent social isolation, which promoted apoptosis. In contrast, swimming exercise suppressed the number of TUNEL-positive cells and Bax and cytochrome $\mathrm{c}$ expression and increased $\mathrm{Bcl}-2$ expression, which inhibited apoptosis.

Exercise increases hippocampal neurogenesis and then enhances learning ability (van Praag et al., 2005). Exercise is an important factor for inhibiting apoptosis (Leasure et al., 2009). In conclusion, social isolation reduced 5-HT expression and promoted apoptosis, leading to anxiety and impaired memory during old age. Swimming exercise improved 5-HT expression and suppressed apoptosis to alleviate anxiety and memory impairment during old age.

\section{CONFLICT OF INTEREST}

No potential conflict of interest relevant to this article was reported.

\section{ACKNOWLEDGMENTS}

This work was supported by the Ministry of Education of the Republic of Korea and the National Research Foundation of Korea (NRF-2016S1A5A2A01027017).

\section{REFERENCES}

Banasr M, Valentine GW, Li XY, Gourley SL, Taylor JR, Duman RS. Chron- ic unpredictable stress decreases cell proliferation in the cerebral cortex of the adult rat. Biol Psychiatry 2007;62:496-504.

Bannerman DM, Sprengel R, Sanderson DJ, McHugh SB, Rawlins JN, Monyer H, Seeburg PH. Hippocampal synaptic plasticity, spatial memory and anxiety. Nat Rev Neurosci 2014;15:181-192.

Daley A. Exercise and depression: a review of reviews. J Clin Psychol Med Settings 2008;15:140-147.

Djordjevic A, Adzic M, Djordjevic J, Radojcic MB. Chronic social isolation is related to both upregulation of plasticity genes and initiation of proapoptotic signaling in Wistar rat hippocampus. J Neural Transm (Vienna) 2009;116:1579-1589.

Dlugosz PJ, Billen LP, Annis MG, Zhu W, Zhang Z, Lin J, Leber B, Andrews DW. Bcl-2 changes conformation to inhibit Bax oligomerization. EMBO J 2006;25:2287-2296.

Goridis C, Rohrer H. Specification of catecholaminergic and serotonergic neurons. Nat Rev Neurosci 2002;3:531-541.

Hall-Lande JA, Eisenberg ME, Christenson SL, Neumark-Sztainer D. Social isolation, psychological health, and protective factors in adolescence. Adolescence 2007;42:265-286.

Hawton A, Green C, Dickens AP, Richards SH, Taylor RS, Edwards R, Greaves CJ, Campbell JL. The impact of social isolation on the health status and health-related quality of life of older people. Qual Life Res 2011;20:57-67.

Huang HJ, Liang KC, Ke HC, Chang YY, Hsieh-Li HM. Long-term social isolation exacerbates the impairment of spatial working memory in APP/PS1 transgenic mice. Brain Res 2011;1371:150-160.

Kuwana T, Newmeyer DD. Bcl-2-family proteins and the role of mitochondria in apoptosis. Curr Opin Cell Biol 2003;15:691-699.

Leasure JL, Decker L. Social isolation prevents exercise-induced proliferation of hippocampal progenitor cells in female rats. Hippocampus 2009;19:907-912.

Lee JM, Ji ES, Kim TW, Kim CJ, Shin MS, Lim BV, Chung YR, Cho YS. Treadmill exercise improves memory function by inhibiting hippocampal apoptosis in pilocarpine-induced epileptic rats. J Exerc Rehabil 2018;14:713-723.

Lee MH, Kim H, Kim SS, Lee TH, Lim BV, Chang HK, Jang MH, Shin MC, Shin MS, Kim CJ. Treadmill exercise suppresses ischemia-induced increment in apoptosis and cell proliferation in hippocampal dentate gyrus of gerbils. Life Sci 2003;73:2455-2465.

López-Toledano MA, Shelanski ML. Neurogenic effect of beta-amyloid peptide in the development of neural stem cells. J Neurosci 2004;24: 5439-5444.

McCormick CM, Thomas CM, Sheridan CS, Nixon F, Flynn JA, Mathews IZ. Social instability stress in adolescent male rats alters hippocampal neurogenesis and produces deficits in spatial location memory in 
adulthood. Hippocampus 2012;22:1300-1312.

Melancon MO, Lorrain D, Dionne IJ. Exercise increases tryptophan availability to the brain in older men age 57-70 years. Med Sci Sports Exerc 2012;44:881-887.

Park SS, Shin MS, Park HS, Kim TW, Kim CJ, Lim BV. Treadmill exercise ameliorates nicotine withdrawal-induced symptoms. J Exerc Rehabil 2019;15:383-391.

Pittenger C, Duman RS. Stress, depression, and neuroplasticity: a convergence of mechanisms. Neuropsychopharmacology 2008;33:88-109.

Ren QG, Gong WG, Wang YJ, Zhou QD, Zhang ZJ. Citalopram attenuates tau hyperphosphorylation and spatial memory deficit induced by social isolation rearing in middle-aged rats. J Mol Neurosci 2015;56: 145-153.

Sargin D, Oliver DK, Lambe EK. Chronic social isolation reduces 5-HT neuronal activity via upregulated SK3 calcium-activated potassium channels. Elife 2016:5:e21416.

Song SH, Jee YS, Ko IG, Lee SW, Sim YJ, Kim DY, Lee SJ, Cho YS. Treadmill exercise and wheel exercise improve motor function by suppress- ing apoptotic neuronal cell death in brain inflammation rats. J Exerc Rehabil 2018;14:911-919.

Staples MC, Somkuwar SS, Mandyam CD. Developmental effects of wheel running on hippocampal glutamate receptor expression in young and mature adult rats. Neuroscience 2015;305:248-256.

Teissier A, Chemiakine A, Inbar B, Bagchi S, Ray RS, Palmiter RD, Dymecki SM, Moore H, Ansorge MS. Activity of Raphé serotonergic neurons controls emotional behaviors. Cell Rep 2015;13:1965-1976.

Upadhyay D, Panduri V, Ghio A, Kamp DW. Particulate matter induces alveolar epithelial cell DNA damage and apoptosis: role of free radicals and the mitochondria. Am J Respir Cell Mol Biol 2003;29:180-187.

van Praag H, Shubert T, Zhao C, Gage FH. Exercise enhances learning and hippocampal neurogenesis in aged mice. Version 2. J Neurosci 2005;25:8680-8685.

Wang M, Bi Y, Zeng S, Liu Y, Shao M, Liu K, Deng Y, Wen G, Sun X, Zeng P, Jing L, Lv Z. Modified Xiaoyao San ameliorates depressive-like behaviors by triggering autophagosome formation to alleviate neuronal apoptosis. Biomed Pharmacother 2019;111:1057-1065. 